\title{
Effects of Pilates Breathing on Chest Expansion, Dyspnoea and Functional Capacity in COPD Patients - An Experimental Study
}

\author{
Gauri Wakde ${ }^{1}$, Prajakta Patil ${ }^{2}$ \\ ${ }^{1}$ MPT Cardiovascular and Respiratory Physiotherapy, Smt. Kashibai Navale College of Physiotherapy, Narhe, \\ Pune, India. \\ ${ }^{2}$ Professor and HOD, Cardiovascular and Respiratory Physiotherapy, Smt. Kashibai Navale College of \\ Physiotherapy, Narhe, Pune, India. \\ Corresponding Author: Gauri Wakde
}

\begin{abstract}
COPD is highly prevalent and not fully reversible. Hence there arises need for finding new significant treatment strategies for treating and preventing COPD. Pilates breathing is growing in both the area of fitness and rehabilitation, but there is scarcely any scientific research on Pilates breathing, particularly in the area related to respiration, with no research done in India. Pilates breathing if proved effective can be of great benefit in curing and rehabilitating respiratory conditions like COPD who present diaphragmatic muscle dysfunction, where it can be of maximum utility. Taking into consideration the progressive acceptance of the Pilates breathing and lack of its studies in COPD, the study was undertaken to study its effectiveness and inculcate it in routine rehabilitation of COPD patients if proven effective.
\end{abstract}

Keywords: Pilates breathing, COPD, Chronic obstructive pulmonary disease, chest expansion, dyspnoea, functional capacity, experimental study.

\section{INTRODUCTION}

Chronic obstructive pulmonary disease (COPD) is a lung disease characterized by chronic obstruction of lung airflow that interferes with normal breathing and is not fully reversible. ${ }^{(1)}$ COPD is the result of a susceptible lung being exposed persistently to triggering environmental stimulus. This is predominantly caused by tobacco smoking, household air pollution, exposure to noxious gases and particles combined with other factors like age, gender, lung status, occupation and duration of exposure. ${ }^{(2,3)}$

COPD is a common preventable and treatable disease which affects people globally. According to WHO, COPD is a silent killer predominantly low and middle income countries like India where the economic impact of COPD is expected to increase to $£ 1.7$ trillion by 2030 . Around 328 million people have COPD worldwide. In next 15 years, COPD is expected to become the leading cause of death worldwide. (4) According to the Global Burden of Disease, COPD is currently the third leading cause of death worldwide, which was not predicted as threat until $2030 .^{(5)}$

India contributes significantly, and has growing percentage of COPD mortality (6) which is estimated to be amongst the highest in the world. COPD was responsible for $75.6 \%$ of chronic respiratory diseases in India as of 2016. The numbers of COPD cases in India were 55.3 million as of 2016. Prevalence rates vary from $2 \%$ to $22 \%$ in men and $1.2 \%$ to $1.9 \%$ in women. COPD takes tolls of $7 \%$ of the population and $3 \%$ loss of disability in India ${ }^{77}$

COPD leads to airflow limitation and the destruction and loss of alveoli, 
terminal bronchioles and surrounding capillary vessels and tissues causing decreased gas transfer capacity. Chronic inflammation leads to narrowing of airways, structural changes and destruction of lung parenchyma which causes loss of alveolar attachments, remaining open during expiration. The reduction of FEV1 and FEV1/FVC can correlate the extent of inflammation, fibrosis and exudates in the airway. The progressive and gradual decline in FEV1 is characteristic of COPD. ${ }^{(8)}$

In COPD, Inspiratory muscles face increased load in expiration but it is affected in inspiration as well. In severe airflow limitation patient initiates inspiration before end expiration which causes air trapping called dynamic hyperinflation. ${ }^{(9,10)}$

Dynamic hyperinflation is caused by mismatch between the demand for respiratory muscle work and the capacity to meet demands. Hyperinflation also increases demand of rib cage and neck muscles decreasing relative contribution of diaphragm to chest wall motion. Respiratory muscle capacity is mainly reduced by lung hyperinflation which is due to incomplete emptying of lungs which leads to air trapping. ${ }^{(11)}$ Air trapping caused due to reduced airflow during exhalation, further results in reduced inspiratory capacity, which may cause dyspnoea on exertion and reduced exercise capacity. Pulmonary hyperinflation shortens and flattens the diaphragm which causes mechanical disadvantage in length tension relationship. ${ }^{(12)}$ leading to reduced chest expansion. ${ }^{(13,14)}$

Hypoxia and hypercapnia result from gaseous exchange abnormality leads to carbon dioxide retention and increased effort of breathing. This further leads to ventilation perfusion mismatch resulting in increased oxygen demand leading to dyspnoea. Dyspnoea is the most common symptom reported in COPD. It acts as a limiting factor during exercise testing and common complaint during daily activities. ${ }^{(15)}$ The Modified Medical Research Council (MMRC) dyspnoea scale is a simple and valid method used for grading the effect of breathlessness on daily activities. ${ }^{(16)}$

Patients with COPD are inactive in daily life due to dyspnoea and reduced chest expansion leading to a downward spiral of symptom-induced inactivity, causing deconditioning and muscle weakness which result in patients spending less time walking and standing leading to a reduction in functional capacity. (17) COPD patients exhibit a shift from type I to type II skeletal muscle fibres, reduced mitochondrial density per fibre bundle and reduced capillary density. Each of these correlates with reduced capacity for aerobic metabolism and ultimately, poorer muscle endurance. (18) Exercise testing is increasingly being used in the functional assessment of COPD patients. A recent review of functional walk tests concluded that the 6MWD is easy to administer, better tolerated and more reflective of activities of daily living than the other tests and it is practically simple. ${ }^{(19)}$

Pilates is a physical fitness system developed in the early 20th century by Joseph Pilates during world war two. Breathing is important aspect in the Pilates method. (20) Complete inhalation and complete exhalation are key to the breathing. (21) Lateral breathing is the important component in Pilates breathing. It is described as a posterior lateral breathing. Lateral breathing emphasizes the lateral expansion of the rib cage while maintaining a consistent inward pull of the deep abdominal muscles by means of active contraction of transverse abdominal and pelvic floor muscles during both inhalation and exhalation. ${ }^{(22)}$

Pilates breathing facilitates correct muscle activation. It focuses on directing the breath into the sides of the ribs rather than the lower stomach so that it helps to keep the abdominals activated when performing exercises. Directing the breath into the sides of the ribs helps to prevent shallow breathing into the upper chest, which otherwise causes the neck and shoulder muscles to tense. 
During inhalation taking a large, deep breath is avoided because this can cause the thoracic spine (the upper and middle back) to extend and the abdomen to protrude, losing the abdominal muscle activation. The breathing in is to inhale and tighten the T-Zone. The pelvic floor contraction encourages the transversus abdominis and deep core muscles.

Pilates focuses on the exhalation as it causes the activation of the abdominal muscles. During exhalation, the transversus abdominis and oblique's contract to increase intra-abdominal pressure and stabilise the spine. Intra-abdominal pressure has influence on spinal stability via production of an extensor moment which exerts a force down on the pelvic floor and up on the diaphragm. ${ }^{(23)}$ A slow exhalation is initiated which enhances the transversus abdominis and oblique activation. Pilates breathing promotes mobility of the ribcage. Separating the ribs with each inhalation pulls every segment of the spine apart which reduces pressure on the discs and helps to develop the length and strength through the torso and also strengthens weak muscles. ${ }^{(24)}$

Considering the burden, there arises need for finding new significant treatment strategies for treating and preventing COPD. The Pilates breathing is growing in both the area of fitness and rehabilitation. (25) However there is hardly any research done on Pilates breathing particularly related to respiration. Effects of Pilates breathing have been studied in obese adults and elderly. Pilates breathing if proved effective can be of great benefit in curing and rehabilitating respiratory conditions like COPD who present diaphragmatic muscle dysfunction, where it can be of maximum utility. Karina $\mathrm{m}$ et $\mathrm{al}{ }^{(21)}$ recommended scope for future study to be undertaken to focus on the effects of Pilates breathing in order to confirm the effects of the intervention. Taking into consideration the progressive acceptance of the Pilates breathing and lack of its studies in COPD the study was undertaken. Studying the effects of Pilates breathing will help to study its effectiveness and inculcate it in routine rehabilitation of COPD patients if proven effective.

\section{MATERIAL AND METHODS}

- Study Design- An Experimental study.

- Study Setting- Pulmonary medicine ward in tertiary care hospital.

- Type of study-Pre and Post intervention design.

- Study Population- Patients with moderate to severe COPD as per GOLD criteria.

- Sample Size-50

- Sample size was calculated using $g$ power based on study by Dimitrova et al (26) using primary outcome measure as 6 minute walk test. To get the effect size of 0.51 in patients with grade 2 to grade 3 (GOLD criteria) at alpha 0.05 and power 95\% the total proposed sample size was 42 .

- The sample size was increased to 50 considering $20 \%$ drop outs.

- Samples were stratified as 25 in each group.

- Sampling Method- Convenient sampling.

Randomisation was done using computer generated randomised table dividing 25 patients in control group and 25 patients in experimental group with age group-45-65 years.

- Study instrument/Tools-Measuring tape, Countdown timer (or stopwatch), two small cones to mark the turnaround points, A chair that can be easily moved along the walking course, Sphygmomanometer, Pulse oximeter, weight cuffs, GOLDs criteria, MMRC scale.

\section{OUTCOME MEASURES-}

Chest expansion- Inch tape (Inches)

$>$ Chest expansion was measured at axillary, nipple and xiphisternal level.

$>$ Chest expansion was noted at maximum inspiration and at maximum expiration.

$>$ Reliability- 0.95-0.97 which indicated excellent test retest reliability. 


\section{MMRC scale}

$>$ Purpose-Dyspnoea

$>$ It is a simple 0-4 graded scale and valid method of categorizing patients with COPD in terms of their disability.

$>$ The patients were showed and explained the MMRC scale and were asked their grade of dyspnoea.

$>$ Reliability-0.82 which indicated good test retest reliability

\section{MINUTE WALK DISTANCE (meters)}

$>$ Purpose-Functional capacity

$>$ Test description- 6 MWD was performed following ATS guidelines.

$>$ Reliability-0.88-0.94 indicates good to excellent test retest reliability.

- Method of Selection of Data subjects-

$>$ Inclusion Criteria-

$>$ Both male and female Patients diagnosed with moderate and severe COPD as per GOLD criteria.

$>$ Age Group- 45 to 65 years.

\section{Exclusion Criteria-}

$>$ Recent abdominal and chest surgeries.

$>$ Patients who are unable to follow commands.

$>$ Severe Neuro-musculoskeletal abnormalities.

$>$ Any other medical condition or disability restricting patient participation.

$>$ Patients doing yoga/pranayama.

\section{Withdrawal criteria-}

$>$ If the vital parameters became unstable the patients were withdrawn from the study.

$>$ Patients who willingly wanted to withdraw from the study were withdrawn.

\section{PROCEDURE}

$>$ Ethical approval from the ethical committee of the institute was obtained.

$>$ Subjects were selected as per inclusion and exclusion criteria.
$>$ Informed written consent of the subjects was taken. All participants received verbal and written information about the study.

$>$ The patients underwent thorough evaluation and treatment maintaining all sterile aseptic precautions.

$>$ Evaluation of-Demographic data like name, age, sex, with contact number was done and anthropometric data such as weight, height, body mass index (BMI) were recorded.

$>$ Clinical assessment and baseline characteristics were checked and recorded.

$>$ The details of patient were filled in Patient assessment sheet and case record form.

$>$ Patients were divided into control group (Group B) and experimental group (Group A) based on computer generated randomised table.

$>$ All the outcome measures (chest expansion, dyspnoea and functional capacity) were assessed before giving the intervention and after completion of 3 weeks of intervention.

$>$ Vitals like HR, RR, BP and SPO2 were recorded pre and post treatment.

$>$ Essential medicines and aerosol treatment was continued for all the patients.

$>$ The patients in control group (Group B) were given conventional treatment (Aerosol treatment + Diaphragmatic breathing + pursed lip breathing + Dyspnoea reliving positions + Strength training of UL and LL).

$>$ Patients in experimental group (Group A) were given Pilates breathing along with conventional treatment.

$>$ Duration of study was 3 weeks. Initial training of 3-4 days were given to the patient to learn the techniques under supervision.

The exercises daily were carried under supervision until the patients were in the hospital. 
$>$ At the time of discharge the patients were given pamphlets with exercise checklist.

$>$ Patients were told to follow the exercises at home and were told to maintain a daily follow up dairy and feedback was taken telephonically.

$>$ Patients were called after 3 weeks for follow up.

> Pre-Post assessment of chest expansion, dyspnoea and 6 MWD was taken. Vitals like HR, BP, RR, and SPO2 were also recorded.

Group A was given Pilates breathing along with conventional treatment.

Pilates breathing - According to the recommendations of Menezes- ${ }^{(22)}$

- Pilates breathing was performed in supine; allowing the respiration to flow, not holding breath at any point.

- Hand placement- Over lower posterior lateral rib cage.

- Patients were instructed to breathe in through nose and breathe out through mouth with lips pursed. In addition to breathing, the abdomen was to be kept pulled in by active contraction of the TrA and pelvic floor muscles.

- Transverse abdominis (TrA) was specifically activated by asking the patient to tighten their stomach as if preparing to be punched in the gut. The patient was asked to hold the pelvic muscles as if trying to hold the urine. The TrA activation was checked by palpating the anterior iliac spine and going to soft tissue right inside of it.

- Frequency of Pilates breathing- 5 times a week

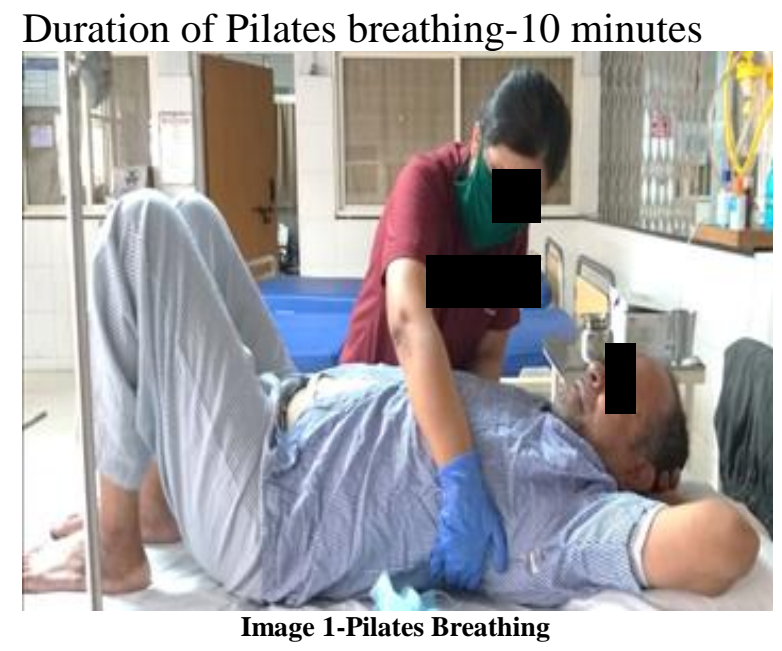

Group B received Conventional treatment which comprised of-

\begin{tabular}{|c|c|c|c|c|c|}
\hline $\begin{array}{l}\text { SR } \\
\text { NO. }\end{array}$ & & MODE & INTENSITY & DURATION & FREQUENCY \\
\hline 1 & Aerosol therapy & Via mask & & $10-15$ minutes & 2-3 times a day \\
\hline 2 & Breathing exercises & $\begin{array}{l}\text { Diaphragmatic breathing. } \\
\text { Pursed lip breathing }\end{array}$ & & 10 minutes & 5 times a week \\
\hline 3 & $\begin{array}{ll}\text { Dyspnoea } & \text { reliving } \\
\text { positions } & \\
\end{array}$ & $\begin{array}{l}\text { Dyspnoea relieving positions } \\
\text { Activity pacing }\end{array}$ & & & 5 times a week \\
\hline 4 & $\begin{array}{l}\text { Upper limb } \\
\text { Mobility and Strength } \\
\text { training }\end{array}$ & $\begin{array}{l}\text { Exercises- } \\
\text { Patient was asked to lift their arms alternately } \\
\text { overhead using a weight cuff }\end{array}$ & $40-60 \%$ & & $\begin{array}{l}10 \text { reps per } \\
\text { session. } \\
5 \text { times a week. }\end{array}$ \\
\hline 5 & $\begin{array}{l}\text { Lower limb Mobility } \\
\text { and Strength training }\end{array}$ & $\begin{array}{l}\text { Dynamic quads, } \\
\text { Squats, Straight leg raise, Step-ups, Sit-to- } \\
\text { stand. Strength training with weight cuffs. }\end{array}$ & $40-60 \%$ & & $\begin{array}{l}10 \text { reps per } \\
\text { session. } \\
5 \text { times a week }\end{array}$ \\
\hline
\end{tabular}

1. Aerosol Therapy-The patient was on essential aerosol therapy.

\section{Breathing exercises-}

1. Diaphragmatic breathing-The patients were placed in the semi fowler position and were instructed to remain relaxed.

Hand placement-One hand was placed on chest and other on stomach.

Technique- As per the standard guidelines.

2. Pursed lip breathing-The patient was sitting with back straight in semi fowler's position, relaxing shoulders as much as possible.

Technique- As per standard guidelines.

3. Dyspnoea reliving positions-The patients were given dyspnoea positions while standing, sitting, and lying as per the guidelines.

\section{Upper Limb mobility and strength training-}


Upper limb mobility of shoulder, elbow and wrist was given to patient.

Strength training was given with weight cuffs, after calculation of 10RM of the patient.

Each exercise was given 10 reps for 5 times a week.

\section{Lower limb mobility and strength training-}

Lower limb mobility of hip, knee and ankle was given to patient.

Strength training was given with weight cuffs, after calculation of 10RM of the patient.

Each exercise was given 10 reps for 5 times a week.

\section{STATISTICAL ANALYSIS}

$>$ The Statistical Package for Social Sciences (version 21; SPSS, Chicago, IL) software was used for statistical analysis and significance level was set at $<0.05$.

$>$ The independent variables were Pilates breathing (experimental group) and conventional treatment (control group) and time (3 weeks intervention).

$>$ Dependent variables were MMRC, 6 MWD and Chest expansion at axillary, nipple and xiphisternal level.

$>$ Mean baseline demographic values were calculated for continuous variables.

$>$ Frequencies were calculated for categorical variables.
$>$ Test for normality: Preliminary analysis (Kolmogorov-Smirnov test) revealed that data was normally distributed $(\mathrm{P}>0.05)$.

$>$ Similarity of baseline measures between groups was assessed using independent $t$ tests.

$>$ As dropouts were very low, further analysis was carried out according to the principles of intention-to-treat analysis.

$>$ Comparisons within and between each group were assessed by $\mathrm{t}$ tests (pre intervention to post intervention) with Bonferroni correction to reduce the chance of type 1 error.

$>$ To determine the magnitude of any treatment effect, Cohens d effect sizes were also calculated for all pair wise comparisons. Effect sizes were rated as small if they were less than 0.40, moderate if between 0.41 and 0.69 , or strong if greater than 0.70 (Cohen, 1988).

\section{RESULTS}

Fifty participants were recruited in the study with 25 in experimental and 25 in control group.

There was drop out of two participants from experimental and three from conventional group.

$>$ Three out of which had difficulty in commuting to hospital.

Two were not keeping well due to health issues.

\section{Gender and Age distribution across the group is presented with graphs:}

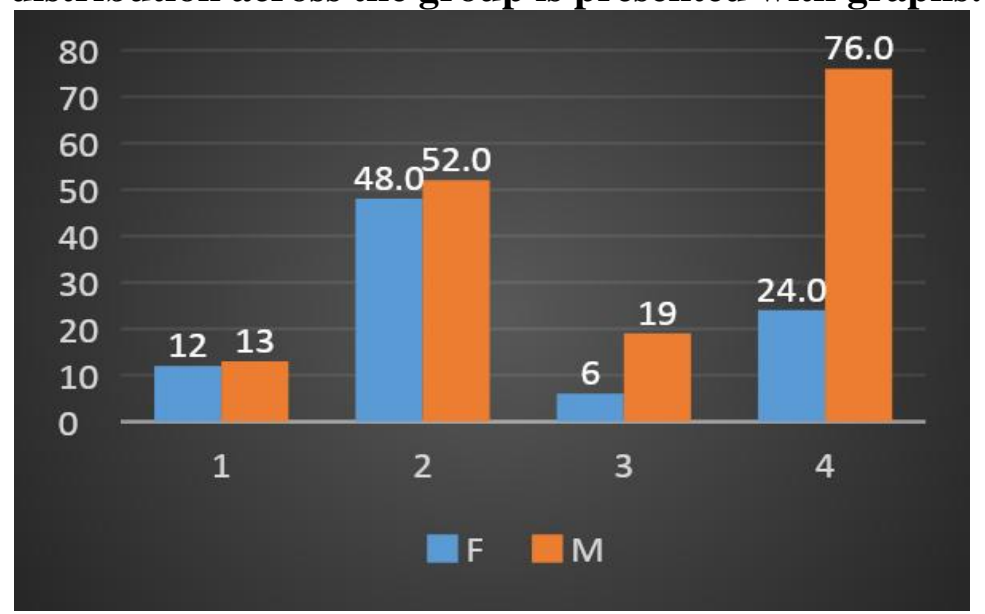

Figure 1: Graph representing gender distribution across the group 
Gauri Wakde et.al. Effects of pilates breathing on chest expansion, dyspnoea and functional capacity in COPD patients - an experimental study.

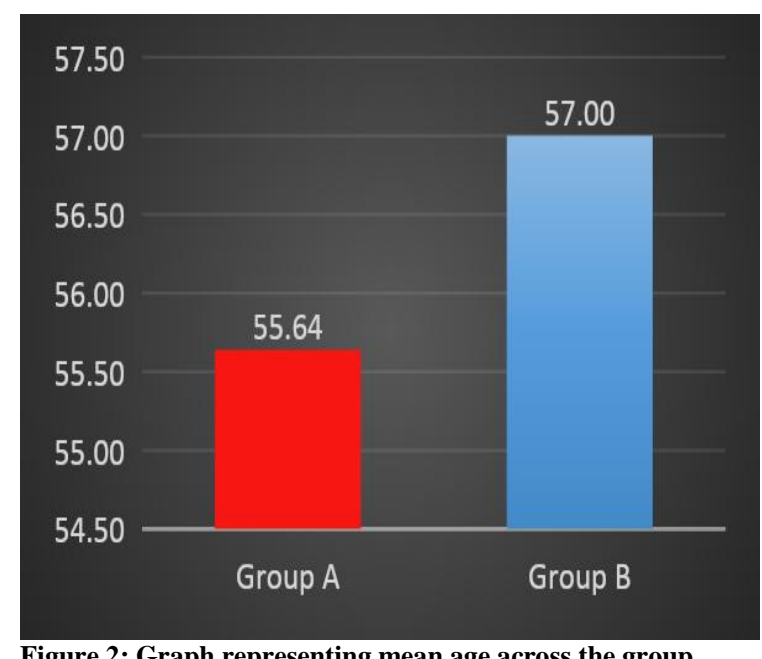

No significant difference in outcome measures was found at baseline between groups $(\mathrm{P}>0.05)$, as shown in Table 1.

Within group analysis demonstrated statistically significant improvements $(\mathrm{p}<$ 0.05) with large effect sizes (Cohen d) between assessment points for all the dependent variables in both groups.

Figure 2: Graph representing mean age across the group

Table 1: Characteristics of participants at baseline

\begin{tabular}{|l|l|l|l|l|l|}
\hline \multicolumn{2}{|c|}{ Table 1: Characteristics of participants at baseline } \\
\hline & Mean A & Std. Deviation & Gean & Std. Deviation & Sig \\
\hline MMRC & 1.96 & 0.73 & 2.08 & 0.64 & 0.48 \\
\hline 6MWD & 362.20 & 56.83 & 347.60 & 79.91 & 0.12 \\
\hline Axillary Level & 0.64 & 0.13 & 0.66 & 0.13 & 0.96 \\
\hline Nipple Level & 0.84 & 0.13 & 0.85 & 0.13 & 0.99 \\
\hline Xiphisternal Level & 0.98 & 0.13 & 1.00 & 0.12 & 0.87 \\
\hline
\end{tabular}

Within group analysis for group $\mathrm{A}$ is presented in the table 2 and for group B presented in the table 3 .

Table 2: Within group analysis for group A

\begin{tabular}{|l|l|l|l|l|l|l|}
\hline & Mean of difference & Std. Deviation & Cohen d & 95\% Confidence Interval & & Sig. (2-tailed) \\
\hline & & & & Lower & Upper & \\
\hline MMRC & 1.04 & 0.20 & 7.35 & 0.96 & 1.12 & 0.00 \\
\hline 6MWD & -55.64 & 17.49 & -4.50 & -62.86 & -48.42 & 0.00 \\
\hline Axillary Level & -0.23 & 0.08 & -4.09 & -0.27 & -0.20 & 0.00 \\
\hline Nipple Level & -0.25 & 0.08 & -4.63 & -0.28 & -0.22 & 0.00 \\
\hline Xiphisternal Level & -0.21 & 0.07 & -4.19 & -0.24 & -0.18 & 0.00 \\
\hline
\end{tabular}

Table 3: Within group analysis for group $B$

\begin{tabular}{|l|l|l|l|l|l|l|}
\hline & Mean of difference & Std. Deviation & Cohen d & 95\% Confidence Interval & & Sig. (2-tailed) \\
\hline & & & & Lower & Upper & \\
\hline MMRC & 1.08 & 0.28 & 5.52 & 0.97 & 1.19 & 0.00 \\
\hline 6MWD & -49.44 & 25.89 & -2.70 & -60.13 & -38.75 & 0.00 \\
\hline Axillary Level & -0.24 & 0.09 & -3.68 & -0.27 & -0.20 & 0.00 \\
\hline Nipple Level & -0.20 & 0.08 & -3.46 & -0.23 & -0.17 & 0.00 \\
\hline Xiphisternal Level & -0.22 & 0.09 & -3.42 & -0.26 & -0.19 & 0.00 \\
\hline
\end{tabular}

Mean and SD for both groups was presented with table 4 and with graphs for MMRC, 6 MWD and chest expansion at all 3 levels

Table 4: Within group changes in mean along with SD for both groups

\begin{tabular}{|l|l|l|l|l|}
\hline & Group A & & Group B & \\
\hline & Pre & Post & Pre & Post \\
\hline MMRC & $1.96(0.73)$ & $0.92(0.70)$ & $2.08(0.64)$ & $1.00(0.58)$ \\
\hline 6 MWD & $362.20(56.83)$ & $417.84(63.51)$ & $347.60(79.91)$ & $397.04(84.49)$ \\
\hline Axillary Level & $0.64(0.13)$ & $0.88(0.13)$ & $0.66(0.13)$ & $0.90(0.13)$ \\
\hline Nipple Level & $0.84(0.13)$ & $1.09(0.15)$ & $0.85(0.13)$ & $1.05(0.14)$ \\
\hline Xiphisternal Level & $0.98(0.13)$ & $1.19(0.13)$ & $1.00(0.12)$ & $1.22(0.16)$ \\
\hline
\end{tabular}


Gauri Wakde et.al. Effects of pilates breathing on chest expansion, dyspnoea and functional capacity in COPD patients - an experimental study.

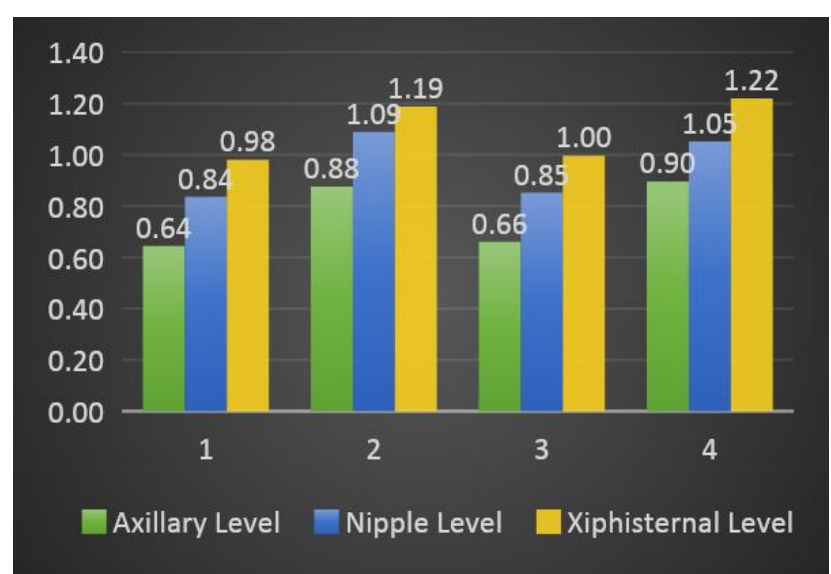

Figure 3- Graph explaining within group changes in mean for chest expansion at all 3 levels for both groups

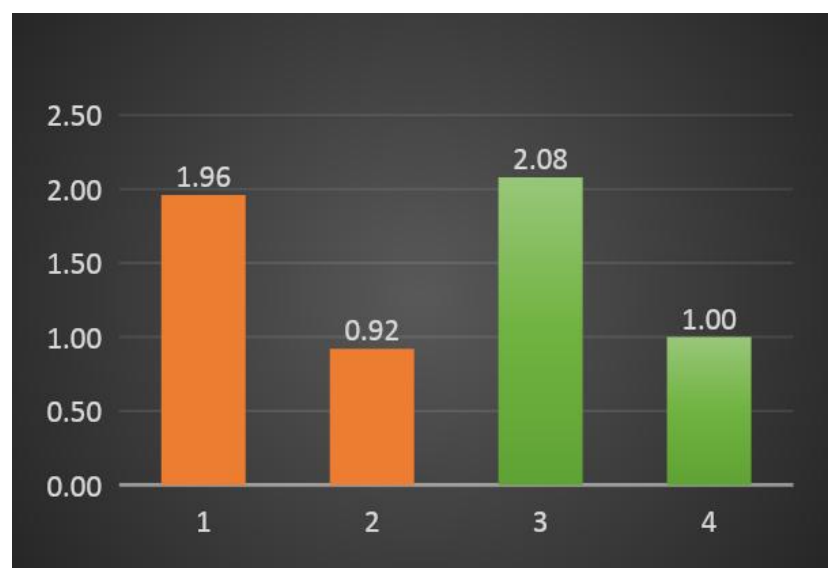

Figure 4- Graph explaining within group changes in mean for MMRC for both groups

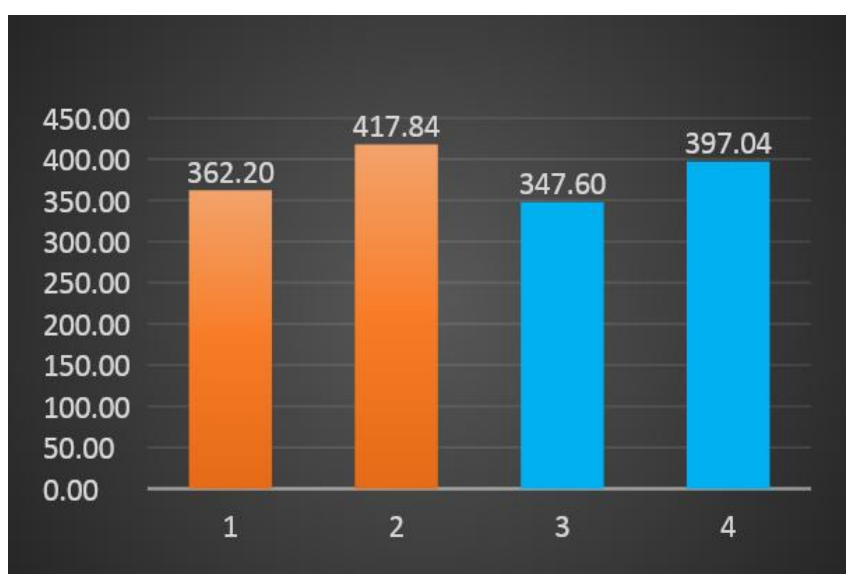

Figure 5-Graph explaining within group changes in mean for 6 MWD for both groups

Between group analysis post significant differences effect size (Cohen d) intervention revealed no significant was not calculated.

difference between both groups in all Between group analysis post outcomes $(\mathrm{P}>0.05)$. As there was no intervention is demonstrated in table 5.

Table 5: Between Group Analysis post Intervention for both groups

\begin{tabular}{|l|l|l|l|l|}
\hline & Mean of difference & 95\% Confidence Interval & Sig. (2-tailed) \\
\hline & & Lower & Upper & \\
\hline MMRC & -0.080 & -0.446 & 0.286 & 0.662 \\
\hline 6MWD & 20.800 & -21.704 & 63.304 & 0.330 \\
\hline Axillary Level & -0.020 & -0.097 & 0.057 & 0.603 \\
\hline Nipple Level & 0.036 & -0.046 & 0.118 & 0.384 \\
\hline Xiphisternal Level & 0.438 & -0.114 & 0.050 & 0.438 \\
\hline
\end{tabular}


Gauri Wakde et.al. Effects of pilates breathing on chest expansion, dyspnoea and functional capacity in COPD patients - an experimental study.

Post intervention mean and SD for both groups was presented in the table 6 and with graphs for MMRC, 6 MWD and chest expansion at all 3 levels

Table 6: Post intervention mean and SD for both groups

\begin{tabular}{|l|l|l|l|l|}
\hline & Group A & & Group B & \\
\hline & Mean & Std. Deviation & Mean & Std. Deviation \\
\hline MMRC & 0.92 & 0.70 & 1.00 & .58 \\
\hline 6 MWD & 417.84 & 63.51 & 397.04 & 84.49 \\
\hline Axillary Level & 0.88 & 0.14 & .90 & 0.13 \\
\hline Nipple Level & 1.09 & 0.15 & 1.05 & 0.14 \\
\hline Xiphisternal Level & 1.19 & 0.13 & 1.22 & 0.16 \\
\hline
\end{tabular}

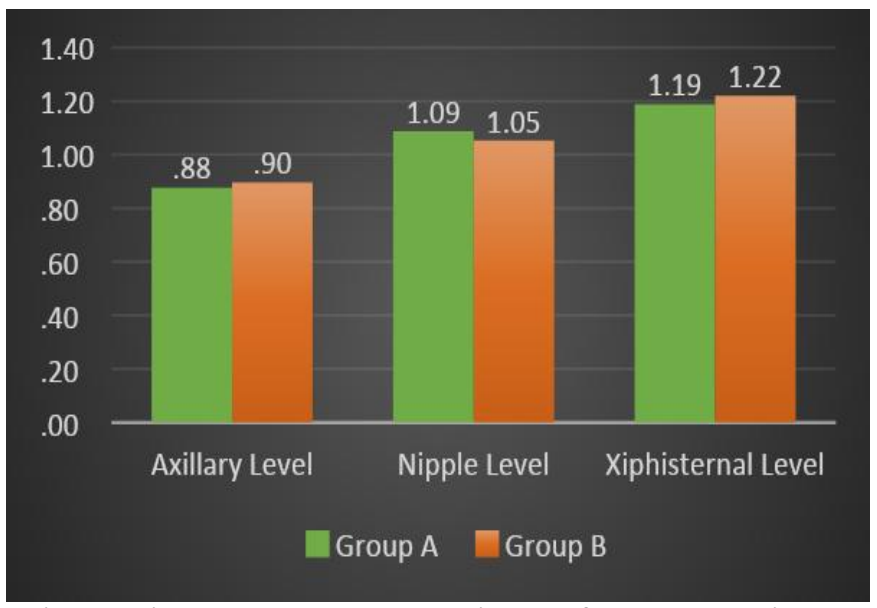

Figure 6- Graph explaining post intervention between group changes in mean for chest expansion at all 3 levels

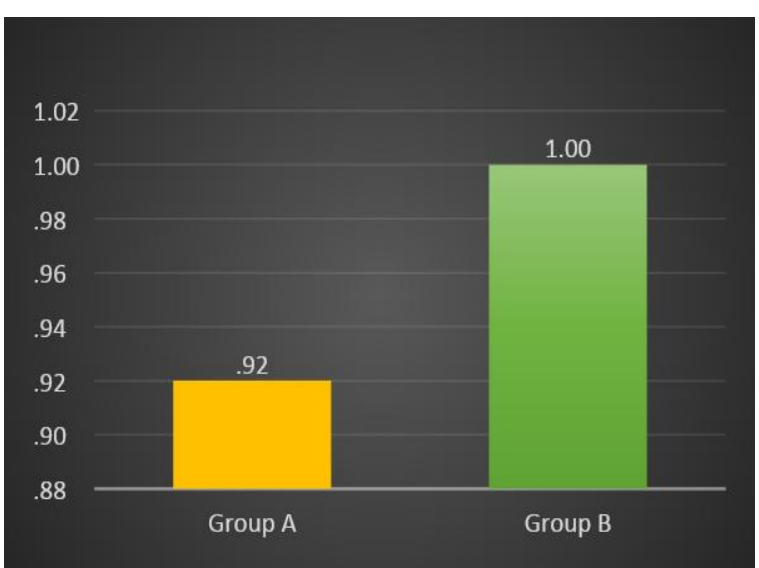

Figure 7- Graph explaining post intervention changes between the groups in mean for MMRC

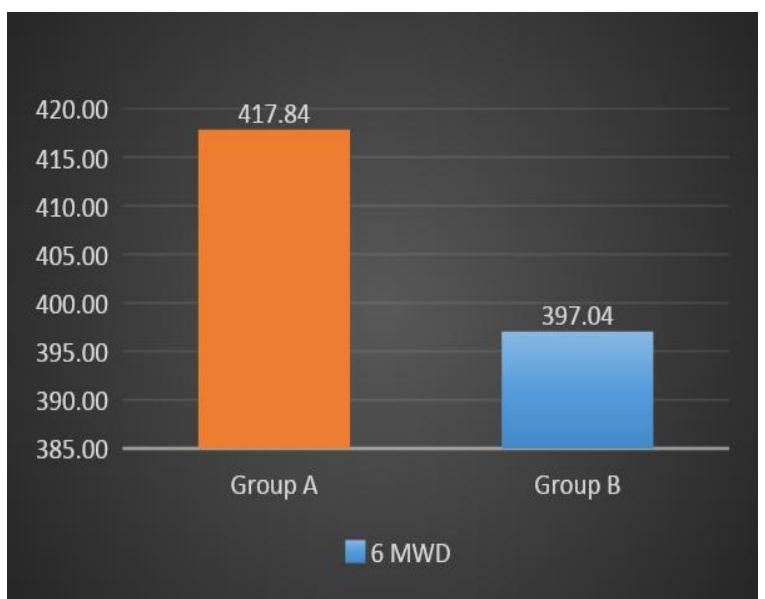

Figure 8- Graph explaining post intervention between group changes in mean for 6 MWD

\section{DISCUSSION}

COPD is a disease that requires multidisciplinary treatment to improve quality of life and increase survival. The evidence till date supports the lasting benefits of breathing exercises and physical exercise in COPD patients.

Airflow limitation in COPD is caused due to abnormalities in airways and destruction of lung parenchyma, which causes reduction of lung elastic recoil leading to hyperinflation, reduced chest wall mobility and subsequently shortness of breath. ${ }^{(27)}$ The physical activities in COPD patients are reduced which further reduce functional capacity. ${ }^{(28)}$

The aim of the study was to study the effects of Pilates breathing on chest expansion, dyspnoea and functional capacity in COPD patients. The study had a control and an experimental group. The patients in control group were given conventional treatment and patients in experimental group were given Pilates breathing along with conventional treatment. 
Amongst the 50 patients, $52 \%$ were men and $48 \%$ women were part of experimental group (group A) while $76 \%$ men and $24 \%$ women attributed to (group B) control group (Figure 1). The higher number of males can be due the higher reported prevalence estimates in men that range from 2 to $22 \%$ as compared to women which is 1.2 to $19 \%$. ${ }^{(29)}$

The mean age in experimental group (group A) was 55.64 while mean age in control group (group B) was 57.00 (Figure 2). The mean age of both the groups supports the study of C. Raherison, P-O Girodet et al which concluded that the incidence of COPD is $15.5 \%$ for those aged $41-50 y r s$; which drastically increases to $23.9 \%$ for those aged 51-60yrs. ${ }^{(30)}$

The specific breathing technique in Pilates is known as lateral breathing, which aims at using the thoracic and ribcage muscles to generate lateral expansion of the ribcage while avoiding expansion of the abdomen. Avoiding the movement of the abdomen provides protection for lumbar region and increases the space for the lungs expansion. ${ }^{(31,32)}$ The diaphragm muscle in Pilates breathing also acts as a stabilizer of the lumbar spine. With active contraction of abdomen and overall stabilizing muscles of the lumbar spine, it is essential to deep breathe to so that the diaphragm muscle and the pelvic floor muscles get involved. ${ }^{(21)}$ Core muscles work by contracting the abdominal muscles, creating higher pressure in abdomen, allowing diaphragm relaxation and carefully controlled upward rise.

The Pilates breathing demarcated statistically significant improvement within the group showing improvement in chest expansion, MMRC and functional capacity (6MWD).

Chest expansion within the experimental group improved significantly at all the three levels. (Figure 3) Pilates breathing promotes activation and recruitment of the abdominal muscles. These muscles are important in both in expiration and inspiration for the facilitation of diaphragmatic action. Pilates breathing leads to positive outcomes in respiratory function due to improvement in diaphragmatic function, which is promoted by the strengthening of the abdominal muscles. ${ }^{(33)}$

According to Barr et al ${ }^{(34)}$ the diaphragm muscle works as the roof of a cylinder of muscles that surround the spine and assist with stability. Maintaining intraabdominal pressure and preventing displacement of the viscera by contraction mainly of the $\operatorname{TrA}$ muscle, is important contribution of diaphragm. Long term benefits can be achieved by the active contraction of the TrA muscle, since it supports descent of diaphragm and provides stabilization of the abdominal compartment. (21) The abdominal distension is prevented by transverse muscles since they promote the movement of lower ribs, give greater support to the diaphragm and provide greater diaphragmatic excursion. (33) According to Janaina Rocha Niehues et al Pilates breathing proved effective in obesity in improving chest expansion. Karina $\mathrm{M}$ et al also concluded that Pilates breathing led to a breathing pattern which increased thoracic expansibility. ${ }^{(21)}$

The above study findings support to findings of our study in which within the group changes in mean at all the levels were noted (Table 2 and Table 4).Hence we can conclude that Pilates breathing led significant improvement in chest expansion. $(\mathrm{p}<0.05)$

Pilates breathing helped to relive dyspnoea which drastically improved the MMRC grade pre and post intervention in experimental group (Figure 4). Dyspnoea is persistent in patients with COPD. In Pilates breathing, contraction of core muscles increases intra-abdominal pressure and reduces intra thoracic pressure by contraction of abdominal muscles which helps to increase airflow. This further improves lung function. ${ }^{(33)}$

Diaphragmatic dysfunction is predominant in COPD. Also the Pilates breathing improves diaphragmatic function by activation of abdominals which provides 
greater chest expansion. This leads to reduced use of accessory muscles, reduces work of breathing and further reduces dyspnoea. Exhalation in Pilates breathing is done with pursed lips. Exhaling through pursed lips helps to splint open the airways and reduce carbon dioxide retention and promote oxygenation. ${ }^{(35,36)}$

Hence it can be concluded that the subjective improvement of dyspnoea in patients with COPD subjects could be result of contraction of core muscles during Pilates breathing which might have led to improvement in diaphragmatic function. Also exhalation through pursed lips might have helped to reduce dyspnoea.

The within the group changes in mean pre intervention to post intervention were achieved (Table 2 and Table 4).Hence Pilates breathing showed significant improvement in MMRC grade subsequently decreasing dyspnoea. $(\mathrm{p}<0.05)$

The functional capacity also drastically improved within the experimental group. Pilates breathing promotes the strengthening of the abdominal muscles. Strengthening abdominals improves diaphragmatic function. Respiratory function improves due to better diaphragmatic function. ${ }^{(33)}$ This further leads to improved exercise capacity and physical function.

Strengthening of core, reduction in dyspnoea and better chest expansion and increase in exercise capacity might have led to improvement in functional capacity in COPD patients (Figure 5).The within group mean distance pre intervention improved (Table 2 and Table 4) So it can be said that Pilates breathing significantly improved 6 MWD, improving functional capacity within the group. $(\mathrm{p}<0.05)$.

The control group also showed significant improvement in chest expansion, dyspnoea and 6 MWD. (Table 2 and Table 4)

Diaphragmatic breathing works for improving diaphragmatic mobility and thereby reducing the deleterious effects of diaphragmatic dysfunction. The abdomen acts as the fulcrum and lifts the lower rib cage and rotates it outwards, reducing dynamic hyperinflation of the rib cage and improving gas exchange and thoracoabdominal motion. In the study by Yamaguti et al, ${ }^{(37)}$ Diaphragmatic breathing training in COPD patients promoted improvement in diaphragm mobility resulting in an improvement in oxygenation and lung function. This could be the probable reason for a significant improvement of chest expansion in control group. (Figure 6)

The dyspnoea reduced in control group. Pursed lip breathing and dyspnoea reliving positions were performed by patients to help reduce dyspnoea. COPD changes in the airways can prevent the appropriate driving pressure and flow of air to maintain an adequate clearance of carbon dioxide due to an increase in airway resistance causing carbon dioxide retention. Purse-lip breathing may be a compensatory mechanism to help splint open the airway and helps to reduce carbon dioxide retention. (36) This further improves lung oxygenation and reduces dyspnoea (Figure 7).

Dyspnoea reliving positions also help to reduce obstruction and improve pulmonary function. The findings of the study match the findings of Nguyen et al which proved pursed lip breathing is helpful for COPD patients.

Reduced exercise capacity is mainly profound in COPD due to muscle dysfunction. Muscle weakness is an important component of impairment and disability in patients with COPD. Upper limb and lower limb strengthening improved strength and subsequently 6 MWD in patients. The findings of this study are similar to findings of Ahmed S Elmorsy et al ${ }^{(37)}$ where it was concluded that the upper limb, lower limb and combined training improved the exercise performance, quality of life and survival in COPD patients (Figure 8). 
Between the groups showed improvement but it was not statistically significant. (Figure 6, Figure 7, Figure 8)

Pilates breathing involves predominantly use of core muscles. It focuses on use of abdominals, diaphragm, trunk and pelvic floor muscles.

There is core muscle weakness, diaphragmatic dysfunction, spinal muscle weakness and pelvic floor weakness in COPD patients. ${ }^{(38,39,40,41,42)}$

Hence in spite of being effective and beneficial and showing statistically significant improvement within the group, Pilates breathing did not show statistically significant improvement between the groups.

\section{CONCLUSION}

Thus it can be concluded from the present study that Pilates breathing is effective in improving chest expansion, reducing dyspnoea and increasing the functional capacity in COPD patients. However it did not prove to be more effective than conventional COPD treatment.

Pilates breathing can be incorporated in rehabilitation of COPD patients along with conventional therapy for earlier and better recovery of patients. Since there were no difficulties addressed by patient while performing Pilates breathing at home during intervention, it can be included in home programme of patients after training.

\section{List of Abbreviations}

COPD Chronic obstructive pulmonary disease

MMRC Modified Medical Research

Council

GOLD Global initiative for Chronic Obstructive Lung Disease
6 MWD
6 Minute Walk Distance

FEV1 Forced expiratory volume

FVC Forced vital capacity

RM Repetition Maximum

Acknowledgement: None

\section{Conflict of Interest: None}

\section{Source of Funding: None}

Ethical Approval: Approved

\section{REFERENCES}

1. COPD; Chronic respiratory diseases, World health organisation.

2. Kim v, Carpo J, ZhaoH Comparison between alternative and classic definition in chronic bronchitis in COPgene, Annuals of American thoracic society; $2015 \quad 12$ (3):332-9

3. Prabu Rajkumar et al, A cross-sectional study on prevalence of chronic obstructive pulmonary disease (COPD) in India: rationale and methods, BMJ, Vol 7 issue 5,2016

4. Lozano R, et al. Global and regional mortality from 235 causes of death for 20 age groups in 1990 and 2010: a systematic analysis for the Global Burden of Disease Study 2010. The Lancet 2012; 380: 20952128

5. Parvaiz A. Koul, Chronic obstructive pulmonary disease: Indian guidelines and the road ahead, Lung India. 2013 Jul-Sep; 30(3): 175-177

6. Sundeep Salvi et al, The burden of chronic respiratory diseases and their heterogenicity across the states of India: the global burden of disease study 1990-2016, Lancet global health, Sep 2018, 1363-1374.

7. Parasuramalu, B.G, Huliraj, N. Kumar, S.P.P and Masthi, Prevalence of Chronic obstructive Pulmonary disease and its association with tobacco smoking and environmental tobacco smoke exposure among rural population. Indian $\mathrm{J}$ Public Health.2014 58;45-49

8. Burgel PR, Nadel JA Epidermal growth factor receptor mediated innate immune response and their role in airway disease. Eur Respir J 2008 Oct; 32(4); 1068-81.

9. Pepe PE, Marini JJ Occult Positive end expiratory pressure in mechanically ventilated patients with airflow obstruction. Am Rev Respir Dis 1982;126:166-70

10. Rossi A, Gottfried SB, Zocchi L et al Measurement of static compliance of total respiratory system in patients with acute ventilatory failure during mechanical ventilation; the effect of intrinsic positive 
end expiratory pressure. Am Rev Respir Dis $1985 ; 131: 672-7$

11. Smith J Bellamare F. Effect of lung volume on in vivo contraction characteristics of human diaphragm. J. Appl Physio 1987;62; 1893-900

12. Klimathianaki M, Vaporidi K, Georgopoulos D. Respiratory muscle dysfunction in COPD; from muscles to cell. Curr Drug target.2011;12:478-488

13. Dangi Ashwini et al,Comparison of Intercostal Stretch Technique Versus Diaphragmatic Breathing on Dyspnoea, Chest Expansion And Functional Capacity in Stable Copd, International Journal of Scientific and Research Publications, Volume 7, Issue 5, May 2017.

14. Rodriguez Roisin, R Drakulovic DA, Roca J, Barbara JA et al, Ventilation Perfusion and Chronic Obstructive Pulmonary Disorder. J App Physio 2009; 106 (6):19028

15. $\mathrm{P}$ Weiner, $\mathrm{R}$ Magadale, $\mathrm{M}$ Beckerman et al. The relationship among inspiratory muscle strength, the perception of dyspnoea and inhaled beta 2 agonist use in patients with asthma. Can Respir J 2002;9(5):307-12

16. Karin Wadell, Katherine A et al, Impact of Pulmonary Rehabilitation on the Major Dimensions of Dyspnoea in COPD, Journal of COPD, 10:425-435, 2013.

17. D. E. O'Donnell, P. Laveneziana. Physiology and consequences of lung hyperinflation in COPD. European Respiratory Review 2006 15: 61-67;

18. Jonathan Singer, Edward H. Yelin, Patricia P. Katz, et al, Respiratory and skeletal muscle strength in COPD: Impact on exercise capacity and lower extremity function; J Cardiopulm Rehabil, Prev.march 2012.

19. Pilates \& Miller 1945, original emphasis

20. Rael isacowitz \& karen clippinger ,Pilates anatomy,2011(Internet)

21. Karina M, Cancelliero-Gaiad1, Daniela Ike et al, Respiratory pattern of diaphragmatic breathing and Pilates breathing in COPD subjects; Braz J Phys Ther. 2014 July-Aug; 18(4):291-299

22. Studio Pilates. The Importance of Breathing in Pilates, 23 July 2018

23. Sung-Tae Kim and Joon He Lee, The effects of Pilates breathing trainings on trunk muscle activation in healthy female subjects: a prospective study. J Phys Ther Sci. 2017 Feb; 29(2): 194-197

24. 16. Ken R. Bracke, Guy G. Brusselle et al, in Mucosal Immunology; (Fourth Edition), 2015.

25. Penelope Latey .Updating the principles of the Pilates method Part 2,Journal of Bodywork and Movement Therapies (2002); 6(2),94-101

26. Antoaneta Dimitrova et al, Physiotherapy in Patients with Chronic Obstructive Pulmonary Disease, 15 Oct 2017; Macedonian Journal of Medical Sciences; 5(6):720-723

27. Morgan AD, Peck DF, Buchannan DR, et al. 1983 Effect of attitudes and beliefs on exercise tolerance in chronic bronchitis. BMJ 286: pp 171-3.

28. Vikram Mohan 2012 Effect of intercostal stretch on pulmonary function parameters among healthy males. EXCLI journal 11: pp 284-290

29. Paryaiz A KOUL, Chronic obstructive pulmonary disease: Indian guidelines and the road ahead .Lung India 2013 Jul-Sep; 30(3): 175-177

30. C. Raherison, P-O Girodet et al, Epidemiology of COPD; European Respiratory Review 2009 18: 213-221

31. Menezes. The complete guide to Joseph $\mathrm{H}$. Pilates' techniques of physical conditioning: applying the principles of body control, Hunter House; 2000.

32. Blount T, McKenzie E. Pilates básico. São Paulo: Manole; 2006

33. Janaina Rocha Niehues; Ana Inés Gonzales; Robson R. et al Pilates Method for Lung Function and Functional Capacity in Obese Adults; Alternative therapies, sep/oct 2015 vol. 21 no. 5

34. Barr KP, Griggs M, Cadby T. Lumbar stabilization: core concepts and current literature: part 1. Am J Phys Med Rehabilitation 2005; 84(6):473-80.

35. Vatwani A. Pursed Lip Breathing Exercise to Reduce Shortness of Breath. Arch Phys Med Rehabil. 2019 Jan;100(1):189-190

36. Sakhaei S, Sadagheyani HE, Zinalpoor $S$ et al. The Impact of Pursed-lips Breathing Maneuver on Cardiac, Respiratory, and Oxygenation Parameters in COPD Patients. Open Access Maced J Med Sci. 2018 Oct 25;6(10):1851-1856

37. Yamaguti WP, Paulin E, Shibao S, Chammas $\mathrm{MC}$ et al trapping: the major 
Gauri Wakde et.al. Effects of pilates breathing on chest expansion, dyspnoea and functional capacity in COPD patients - an experimental study.

factor limiting diaphragm mobility in chronic obstructive pulmonary disease patients. Respirology. 2008; 13(1):138-44

38. Ahmed S Elmorsy et al, Effect of upper limb, lower limb and combined training on exercise performance, quality of life and survival in COPD; Egyptian Journal of Chest Diseases and Tuberculosis, Volume 61, Issue 3, July 2012, Pages 89-93

39. Priya Deshmukh, Rachna Arora, Comparison Of Core Muscle Strength In Chronic Obstructive Pulmonary Disease And In Age, Gender and BMI Matched Healthy Individuals.

40. Ki-song Kim, Min-kwang Byun, et all, Effects of breathing manoeuvre and sitting posture on muscle activity in inspiratory accessory muscles in patients with chronic obstructive pulmonary disease
Multidisciplinary Respiratory Medicine 2012;7:9.

41. Kjensli, J. A. Falch, M. Ryg, T. Blenk, G et al, Prevalence of vertebral deformities in COPD patients: relationship to disease severity. European Respiratory Journal 2009 33: 1018-1024

42. Button BM, Sherburn M, Chase J, et al. Pelvic floor muscle dysfunction. 2005

How to cite this article: Wakde G, Patil P. Effects of pilates breathing on chest expansion, dyspnoea and functional capacity in COPD patients - an experimental study. International Journal of Research and Review. 2021; 8(5): 249-262. DOI: https://doi.org/10.52403/ijrr. 20210533 